\title{
Endoscopic ultrasound-guided biliary drainage with a novel fine-gauge balloon catheter: simplified technique using a coaxial guidewire
}

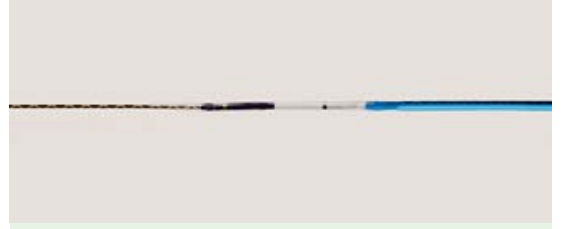

Fig. 1 The tip of the balloon catheter is only $3 \mathrm{Fr}$ in diameter, tapered, and coaxial with the 0.025 -in guidewire.

For performance of endoscopic ultrasound-guided biliary drainage (EUS-BD), such as with EUS-guided hepaticogastrostomy (EUS-HG) [1,2], the fistula must be dilated so that the stent delivery system can be inserted [3]. We demonstrate herein a simplified technique for performing EUS-BD with a novel fine-gauge balloon catheter (REN Biliary Dilation Catheter; Kaneka Corporation, Osaka, Japan).

This balloon catheter is designed for use coaxially with a 0.025 -inch guidewire ( $\bullet$ Fig. 1), having a lumen through which the guidewire can be advanced. In addition the catheter tip is only $3 \mathrm{Fr}$ in diameter and is tapered. For an EUS-BD procedure, the dilation device must have a fine gauge and adequate stiffness to dilate the fistula. After the bile duct has been punctured with a 19-gauge aspiration needle, this balloon catheter can easily be inserted without any dilation devices. Therefore, the risk for bile leakage from the fistula may be decreased because no device exchange is needed and the procedure time is reduced.

A 77-year-old woman admitted for obstructive jaundice due to pancreatic cancer underwent distal gastric resection with a Roux-en-Y procedure. EUS-HG was selected as the biliary drainage method. First, we punctured the left intrahepatic bile duct with a 19-gauge aspiration needle ( Fig.2a) and injected contrast medium. The lower biliary duct was obstructed. Then, we inserted the stiff 0.025-inch guidewire ( Fig. $\mathbf{2}$ b). After the guidewire had been inserted into the intestine, the novel balloon catheter was easily and smoothly inserted into the intestine across the stricture site and
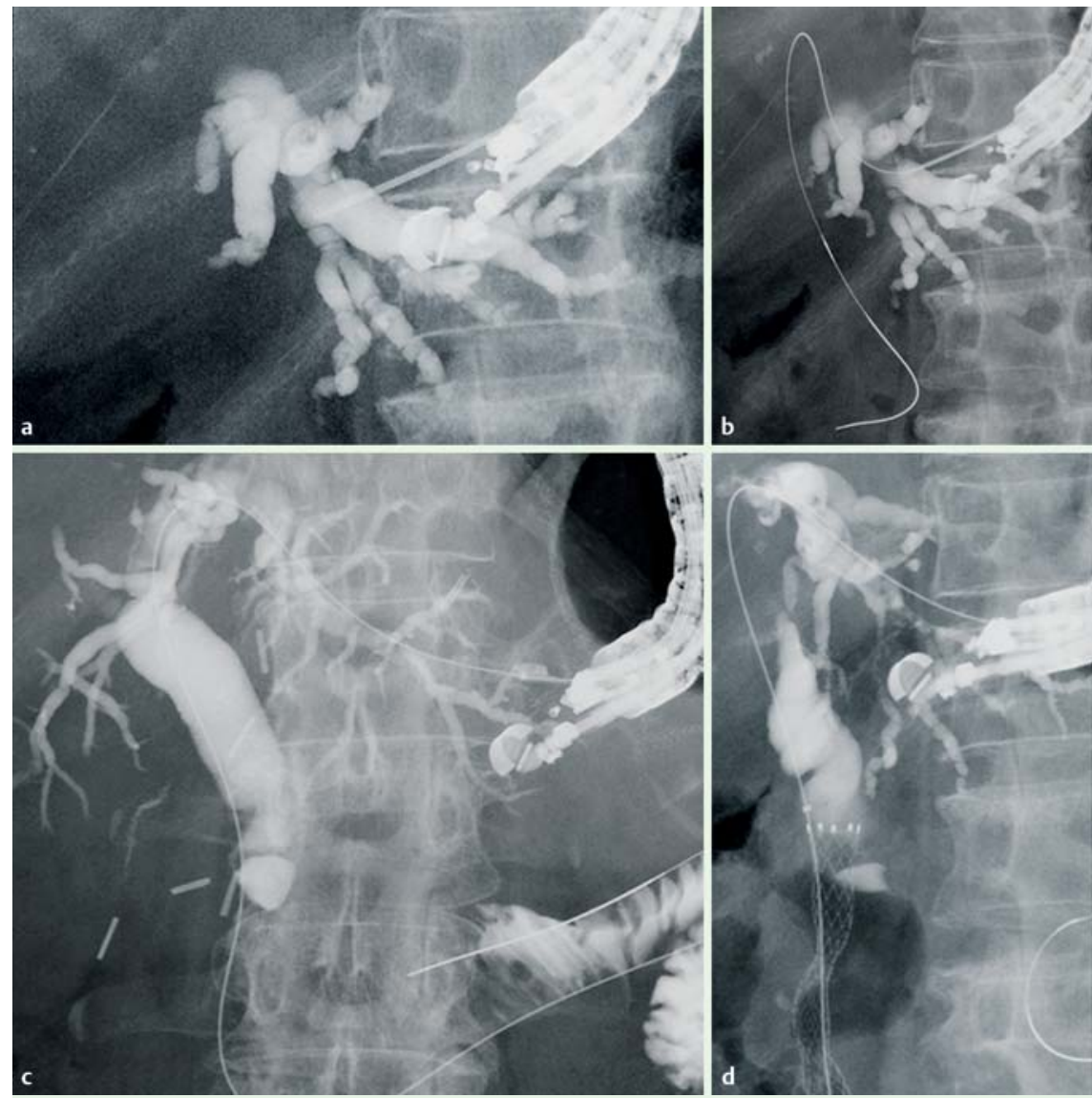

Fig.2 In a 77-year-old woman with obstructive jaundice due to pancreatic cancer, hepaticogastrostomy is selected as the biliary drainage method. a The intrahepatic bile duct is punctured with a 19-gauge aspiration needle. $\mathbf{b}$ The guidewire is inserted into the biliary tract. $\mathbf{c}$ The stomach wall is dilated with the novel balloon catheter. $\mathbf{d}$ A fully covered metallic stent is placed in antegrade fashion.

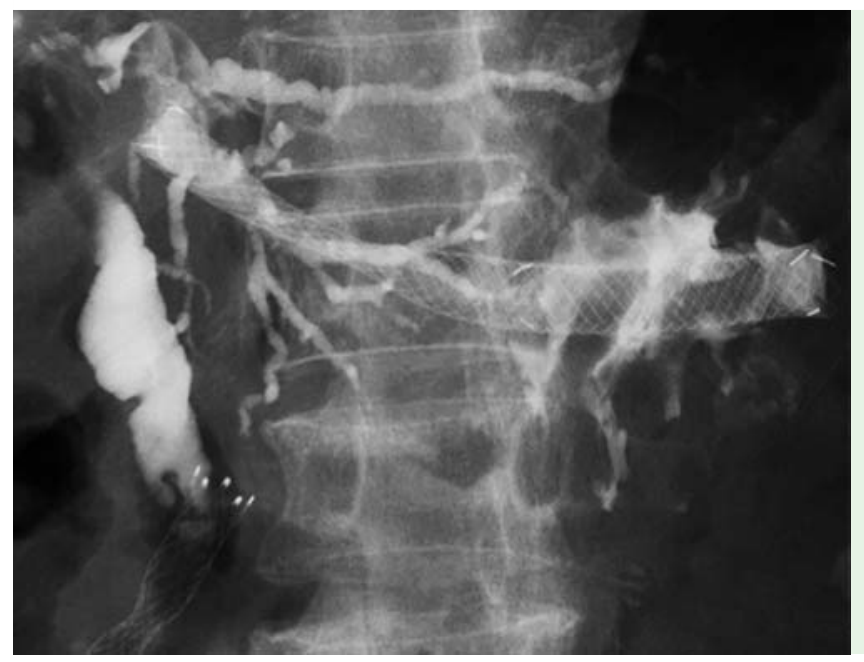

Fig. 3 Endoscopic ultrasound-guided hepaticogastrostomy is completed. 


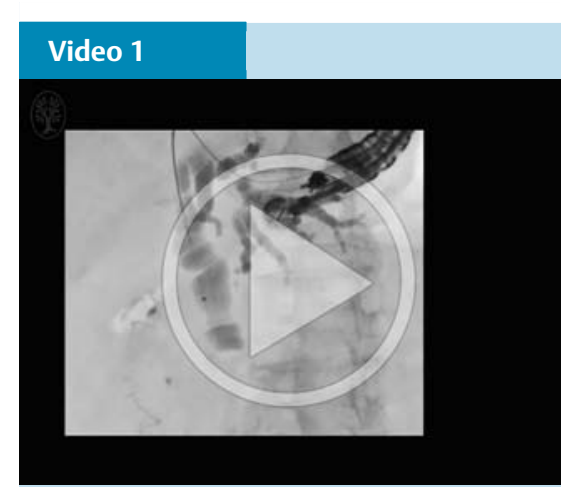

The intrahepatic bile duct is punctured with a 19-gauge aspiration needle, and contrast medium is injected. The guidewire is inserted, and then the novel balloon catheter is easily inserted. After balloon dilation, antegrade stent placement is performed. Finally, endoscopic ultrasound-guided hepaticogastrostomy is completed. used to dilate the intrahepatic bile duct and stomach wall ( $\bullet$ Fig.2c). A metallic stent was placed in antegrade fashion

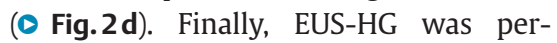
formed ( $\bullet$ Fig. 3, $\bullet$ Video 1 ). No adverse events occurred, and the procedure time was only 21 minutes.

This novel balloon catheter facilitates fistula dilation in EUS-BD procedures, and the procedure time may be reduced.

Endoscopy_UCTN_Code_TTT_1AT_2AB

Competing interests: None

Takeshi Ogura, Wataru Takagi, Saori Onda, Daisuke Masuda, Toshihisa Takeuchi, Shinya Fukunishi, Kazuhide Higuchi

2nd Department of Internal Medicine, Osaka Medical College, Osaka, Japan

\section{References}

1 Park do H. Endoscopic ultrasonographyguided hepaticogastrostomy. Gastrointest Endosc Clin N Am 2012; 22: 271 - 280, ix

2 Ogura T, Masuda D, Imoto $A$ et al. EUS-guided hepaticogastrostomy combined with finegauge antegrade stenting: a pilot study. Endoscopy 2014; 46: 416-421

3 Park do H, Jeong SU, Lee BU et al. EUS-guided biliary drainage with transluminal stenting after failed ERCP: predictors of adverse events and long-term results. Gastrointest Endosc 2011; 74: 1276-1284

\section{Bibliography}

DOI http://dx.doi.org/

10.1055/s-0034-1393380

Endoscopy 2015; 47: E573-E574

(c) Georg Thieme Verlag KG

Stuttgart · New York

ISSN 0013-726X

Corresponding author

Takeshi Ogura, MD

2nd Department of Internal Medicine

Osaka Medical College

2-7 Daigakuchou

Takatsukishi

Osaka 569-8686

Japan

Fax: +81-726846532

oguratakeshi0411@yahoo.co.jp 